\title{
Haemaphysalis japonica, Haemaphysalis jezoensis and "Haemaphysalis douglasi" (Acari: Ixodidae): Which tick is distributed in Hokkaido?
}

\author{
Minoru NaKaO $\left.{ }^{*}, 1\right)$ and Takuya ITO ${ }^{2)}$ \\ * Corresponding author: Department of Parasitology, Asahikawa Medical University, Asahikawa, Hokkaido \\ 078-8510, Japan (E-mail: nakao@asahikawa-med.ac.jp) \\ ${ }^{1)}$ Department of Parasitology, Asahikawa Medical University, Asahikawa, Hokkaido 078-8510, Japan \\ ${ }^{2)}$ Department of Infectious Diseases, Hokkaido Institute of Public Health, Sapporo, Hokkaido 060-0819, Japan
}

(Received: 15 November 2013; Accepted: 1 February 2014)

\begin{abstract}
As a general view, the ixodid tick Haemaphysalis japonica is distributed in Japan (Kyushu, Honshu and Hokkaido islands) and in a continental area including Russia Primorsky Krai, eastern China and the Korean Peninsula. The continental population is treated as a subspecies, Haemaphysalis japonica douglasi. The Hokkaido population was once named Haemaphysalis jezoensis, but synonymized with $H$. japonica. An irregular taxonomic revision, however, elevated $H$. japonica douglasi to specific rank for the continental and Hokkaido populations. The resultant "Haemaphysalis douglasi" sensu Kitaoka is obviously invalid because of the lack of taxonomic literatures about its specific status. Even in the present time, the invalid name has yet been used for tick identification in Hokkaido. In this study, nucleotide sequences of mitochondrial DNA (large subunit ribosomal RNA gene) and nuclear DNA (internal transcribed spacer 2) were compared between adult samples of $H$. japonica from Honshu and those of "H. douglasi" from Hokkaido. The target sequences of the Hokkaido samples were completely identical with those of the Honshu samples, indicating that the elevation of $H$. japonica douglasi to specific rank or the resurrection of $H$. jezoensis is an inadequate treatment. The result clearly shows that $H$. japonica is distributed in Hokkaido.
\end{abstract}

Key words: Haemaphysalis japonica, Hokkaido, Honshu, genetic profile

\section{INTRODUCTION}

Animal scientific names of new species and new combinations can be used only after publications based on rules of the International Code of Zoological Nomenclature. An irregular elevation of subspecies to specific rank and a continuous use of the resultant invalid name cause a lot of confusion on taxonomy. We report here a problematic issue on the taxonomy of ixodid ticks in Hokkaido, Japan.

As a general view, Haemaphysalis japonica Warburton, 1908 (the Japanese name: Yamato Chimadani) is distributed in Japan (Kyushu, Honshu and Hokkaido islands) and in a continental area including Russia Primorsky Krai, eastern China and the Korean Peninsula (Yamaguti et al., 1971; Kolonin, 1978; Teng and Jiang, 1991). Large and mediumsized mammals serve as hosts for the adult ticks (Pomerantzev, 1950). Historically, Warburton (1908) briefly described this species based on male specimens kept at the British Museum. The additional information was given by a monograph of Nuttall and Warburton (1915). Types of this species were collected from the Japanese serow Nemorhaedus crispus [sic] at Hondo (probably a city of Kyushu island), Japan, by the Duke of Bedford's collector. The monograph also included the description of Haemaphysalis japonica var. douglasi. This variety was made using male specimens from roe deer in northern China, based on the smaller body size and slightly changed morphology of scutum and palps (Nuttall and Warburton, 1915). Pomerantzev (1950) treated this variety as a subspecies (i.e. Haemaphysalis japonica douglasi). The subspecies is distributed in the above-mentioned continental area, whereas the nominotypical subspecies in Japan.

Another related species, Haemaphysalis jezoensis Ogura and Takada, 1927, was found from cattle and horses in Hokkaido. However, the original description included no comments on H. japonica (Ogura and Takada, 1927). Long afterwards $H$. jezoensis indigenous to Hokkaido was synonymized with $H$. japonica (Yamaguti et al., 1971), but there is a little possibility that H. jezoensis is a cryptic species. To differentiate immature stages of Haemaphysalis ticks in Japan, Kitaoka (1985) showed keys to the species, together with an irregular taxonomic revision. He mentioned that $H$. japonica in Hokkaido was identical to the continental subspecies in Russia. Based on morphological differences from $H$. japonica in Honshu, $H$. japonica douglasi was elevated to specific rank even though the lack of taxonomic descriptions. Consequently, the revision limits the distributional range of $H$. japonica to Honshu and Kyushu islands. At the present time, however, the new combination "Haemaphysalis douglasi" sensu Kitaoka, 1985 is obviously invalid because there are still no literatures to define its specific status based on 
morphological, ecological and genetic considerations. Types of "H. douglasi" also remain to be specified. The most recent list of valid species names of ixodid ticks in the world ignores "H. douglasi" (Guglielmone et al., 2010).

A picture book of ticks and mites in Japan (Takada, 1990) introduced the name of " $H$. douglasi" (the Japanese name: Dagurasu Chimadani) into ordinary researchers. After that, scientific papers using the invalid name have been gradually increased (Takada et al., 1998; Fujita et al., 2000; Yoshimoto et al., 2009; Yokoyama et al., 2012; Tagawa et al., 2013), but a few researchers used names of $H$. japonica and $H$. jezoensis for the same organism (Ozawa and Kadosaki, 1996; Ito and Takahashi, 2001). Such a confused and strange situation is still continuing, particularly in Hokkaido. In this study, mitochondrial DNA (mtDNA) and nuclear DNA (nDNA) were compared between $H$. japonica and "H. douglasi" to clarify their specific status.

\section{Materials And Methods}

One female tick and one male tick of $H$. japonica from Honshu (collected in Matsumoto, Nagano prefecture) and three female ticks of " $H$. douglasi" from Hokkaido (collected in three places of Funano, Kitami, and Takinoue) were used for molecular comparison. One of the authors (M.N.) judged that the Hokkaido samples were morphologically indistinguishable from $H$. japonica, based on the diagnosis of Yamaguti et al. (1971). Ticks of Haemaphysalis flava Neumann, 1897 from Ome, Tokyo, Haemaphysalis megaspinosa Saito, 1969 from Samani, Hokkaido and Haemaphysalis longicornis Neumann, 1901 from Ome, Tokyo were added as controls. One female tick from each control species was used for analysis. All of the ticks were collected by flagging vegetation during spring seasons of 2012 and 2013. Fragments of mtDNA (large subunit ribosomal RNA gene, $r r n L$ ) and nDNA (internal transcribed spacer 2, ITS2) were amplified from genomic DNA by polymerase chain reaction (PCR) using published primers (Tian et al., 2011; Barker,
1998). The genomic DNA was extracted from ticks using QIAGEN DNeasy blood and tissue kit, and the PCR was run using TaKaRa ExTaq DNA polymerase. Both methods were carried out as recommended by the manufacturers. Nucleotide sequences of PCR amplicons were directly read using BigDye terminator cycle sequencing kit and Applied Biosystems 3500 genetic analyzer. PCR primers were also used for sequencing primers. Primer walking was done in ITS2 sequences. Nucleotide alignments were made by MAFFT (Katoh et al., 2013). Pairwise divergence values and neighborjoining trees were calculated by MEGA5 (Tamura et al., 2011) using K2 parameter with a gamma setting of 0.5 .

\section{Results AND Discussion}

Lengths of nucleotide sequences determined in this study were 404-409 bp of mitochondrial $\mathrm{rrnL}$ and 1488-1846 bp of nuclear ITS2. These were deposited in DDBJ/EMBL/GenBank databases under the accession numbers AB861936-43. The rrnL sequences of three individual "H. douglasi" from Hokkaido were completely identical with those of two individual $H$. japonica from Nagano, showing that the Hokkaido individuals should be identified as $H$. japonica. The complete identity was also confirmed even in highly variable ITS2 region. Concordant results of the mtDNA and nDNA markers suggest that regional genetic variety of $H$. japonica is non-existent or very small in Japan. The number of ticks examined in this study is very small, but the complete sequence agreement between samples from the two distantly located areas is considered sufficient to confirm "H. douglasi" as H. japonica. Moreover, the

Table 1. Pairwise divergence values of mitochondrial DNA $(r r n L)$ and nuclear DNA (ITS2) between H. japonica and other ticks.

\begin{tabular}{lll}
\hline \hline H. japonica versus & $r r n L$ & ITS2 \\
\hline "H. douglasi" & 0.000 & 0.000 \\
H. megaspinosa & 0.002 & 0.018 \\
H. flava & 0.067 & 0.021 \\
H. longicornis & 0.181 & 0.299 \\
\hline
\end{tabular}
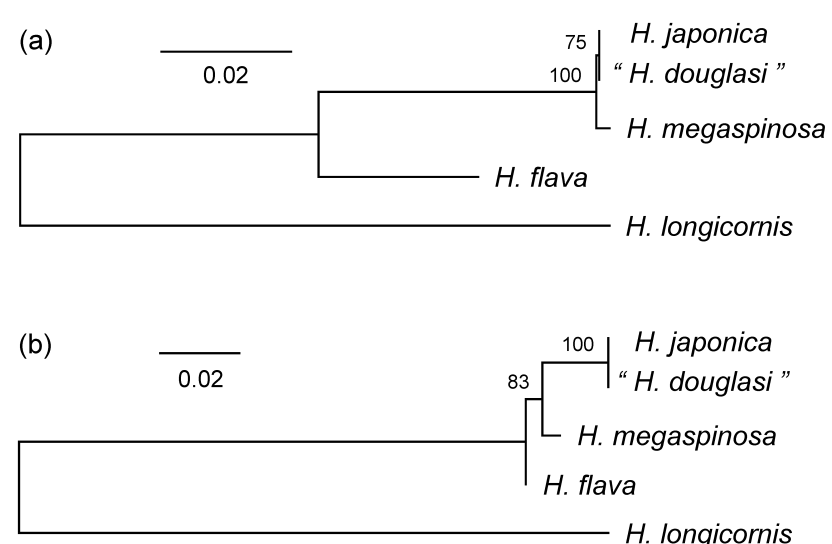

Fig. 1. Neighbor-joinig trees of Haemaphysalis ticks used in this study. Values on the tree nodes are bootstrap proportions (\%) in 500 replicates. Scale bars (divergence of 0.02) are shown. (a) Phylogram from mitochondrial rrnL. (b) Phylogram from nuclear ITS2. 
genetic identity between $H$. japonica and " $H$. douglasi" has been independently confirmed by other researchers (Takano and Kawabata, personal communication). Genetic divergence values between $H$. japonica and other species are shown in Table 1. Unrooted trees illustrate the relationship of these species (Fig. 1). Among limited number of taxa, $H$. japonica was most related to $H$. megaspinosa, and secondarily to $H$. flava. These close genetic similarities indicate a possibility that an evolutionarily young group exists in species of the Far Eastern Haemaphysalis. In particular, the present data raise a hypothesis that $H$. megaspinosa may be conspecific to $H$. japonica, although $H$. megaspinosa has distinctive morphological features (Saito, 1969).

In conclusion, the elevation of $H$. japonica douglasi to specific rank or the resurrection of $H$. jezoensis is an inadequate taxonomic treatment. The present result clearly demonstrates that $H$. japonica is distributed in Hokkaido, as already shown by Yamaguti et al. (1971). Morphological differences observed in the Hokkaido population of " $H$. douglasi" (Kitaoka 1985; Takada 1990) seem to be intraspecific variations of $H$. japonica. The Japanese name "Dagurasu Chimadani" for " $H$. douglasi" also should be eliminated. Further studies are necessary to compare genetic profiles between Hokkaido and Russian populations of $H$. japonica.

\section{ACKNOWLEDGEMENTS}

Special thanks are due to Ai Takano (Yamaguchi University) and Hiroki Kawabata (National Institute of Infectious Diseases) for supplying their unpublished phylogenetic tree of Japanese ticks.

\section{REFERENCES}

Barker, S. C. 1998. Distinguishing species and populations of rhipicephaline ticks with its 2 ribosomal RNA. J. Parasitol., 84: 887-892.

Fujita, H., Takada, N., Isogai, E., Watanabe, Y. and Ito, T. 2000. Isolation of spotted fever group rickettsiae from Ixodes persulcatus ticks in the central part of Hokkaido, Japan. Med. Entomol. Zool., 51: 55-58.

Guglielmone, A. A., Robbins, R. G., Apanaskevich, D. A., Petney, T. N., Estrada-Pena, A., Horak, I. G., Shao, R. F. and Barker, S. C. 2010. The Argasidae, Ixodidae and Nuttalliellidae (Acari: Ixodida) of the world: a list of valid species names. Zootaxa, 2528: 1-28.

Ito, T. and Takahashi, K. 2001. Study on the exoparasites of sika deer Cervus nippon in Hokkaido. Final study report on the preservation and control of Hokkaido sika deer, 1996-2000. Hokkaido government. 203-216. (in Japanese)

Katoh, K. and Standley, D. M. 2013. MAFFT multiple sequence alignment software version 7: improvements in performance and usability. Mol. Biol. Evol., 30: 772-780.

Kitaoka, S. 1985. Keys to the species in immature stages of the
Japanese Haemaphysalis ticks (Ixodidae). Bull. Natl. Inst. Anim. Health, 88: 49-63. (in Japanese)

Kolonin, G. V. 1978. World distribution of ixodid ticks (genus Haemaphysalis). Moscow, Nauka, 70 p. (In Russian)

Nuttall, G. H. F. and Warburton, C. 1915. Ticks, a monograph of the Ixodoidea. Part III. The genus Haemaphysalis. p 349-550. Cambr. Univ. Press.

Ogura, K., Takada, K. 1927. The ticks parasitic on cattle and horses in Hokkaido, Japan. J. ColI. Agric., Hokkaido Imp. Univ. 18: 199-206.

Ozawa, Y. and Kadosaki, M. 1996. Seasonal dynamics of the population of the five species of ticks collected from the brown bear and its habitat in Hokkaido. J. Jpn. Wildl. Res. Soc, 22: 29-42. (in Japanese)

Pomerantzev, B. I. 1950. Fauna of the USSR. Arachnida. 4(2). Ixodid Ticks (Ixodidae). Zool. Inst. Acad. Sci. USSR. New Series No. 41, Moscow. 224 p. (English translation published by Amer. Inst. Biol. Sci., Washington)

Saito, Y. 1969. Studies on ixodid ticks. X. Haemaphysalis megaspinosa n. sp. (Ixodoidea, Ixodidae) from Kanagawa Prefecture, Japan. Acta Med. Biol. (Niigata), 17: 87-96.

Tagawa, M., Ybañez, A. P., Matsumoto, K., Yokoyama, N. and Inokuma, H. 2013. Interference between Theileria orientalis and hemotropic Mycoplasma spp. (hemoplasmas) in grazing cattle. Vet. Parasitol., 195: 165-168.

Takada, N. 1990. Byougen Danirui Zufu. Kinpodou, Kyoto, 216 p. (in Japanese)

Takada, N., Ishiguro, F., Fujita, H., Wang, H.-P., Wang, J.-C. and Masuzawa, T. 1998. Lyme disease spirochetes in ticks from northeastern China. J. Parasitol., 84: 499-504.

Tamura, K., Peterson, D., Peterson, N., Stecher, G., Nei, M. and Kumar, S. 2011. MEGA5: molecular evolutionary genetics analysis using maximum likelihood, evolutionary distance, and maximum parsimony methods. Mol. Biol. Evol., 28: 2731-2739.

Teng, K. F. and Jiang, Z. J. 1991. Acari: Ixodidae. Economic Insect Fauna of China. Science Press, Beijing, Fasc. 39, 359 p.

Tian, Z., Liu, G., Xie, J., Yin, H., Luo, J., Zhang, L., Zhang, P. and Luo, J. 2011. Discrimination between Haemaphysalis longicornis and $H$. qinghaiensis based on the partial $16 \mathrm{~S}$ rDNA and the second internal transcribed spacer (ITS-2). Exp. Appl. Acarol., 54: 165172.

Warburton, C. 1908. On some new and obscure species of the genus Haemaphysalis of the Ixodidae. Proc. Cambr. Philos. Soc., 14: 508-519.

Yamaguti, N., Tipton, V. J., Keegan, H. L. and Toshioka, S. 1971. Ticks of Japan, Korea, and the Ryukyu islands. Brighan Young Univ. Sci. Bull. Biol. Ser., 15: 1-226.

Yokoyama, N., Sivakumar, T., Ota, N., Igarashi, I., Nakamura, Y., Yamashina, H., Matsui, S., Fukumoto, N., Hata, H., Kondo, S., Oshiro, M., Zakimi, S., Kuroda, Y., Kojima, N., Matsumoto, K. and Inokuma, H. 2012. Genetic diversity of Theileria orientalis in tick vectors detected in Hokkaido and Okinawa, Japan. Infect. Genet. Evol., 12: 1669-1675.

Yoshimoto, K., Matsuyama, Y., Matsuda, H., Sakamoto, L., Matsumoto, K., Yokoyama, N. and Inokuma, H. 2010. Detection of Anaplasma bovis and Anaplasma phagocytophilum DNA from Haemaphysalis megaspinosa in Hokkaido, Japan. Vet. Parasitol., 168: $170-172$. 